\title{
Environmental Effect of Tidal Bore Propagation in Kampar River
}

\author{
Mubarak $^{1, *}$, Albertus Sulaiman ${ }^{2}$, and Efriyeldi ${ }^{1}$ \\ ${ }^{1}$ Department of Marine Sciences, Universitas Riau, Indonesia \\ ${ }^{2}$ Geostech Laboratory BPPT, Kawasan Puspiptek Serpong, Indonesia.
}

\begin{abstract}
The environmental effect of the tidal bore propagation in kampar river is investigated. Propagation of the tidal bore satisfy Korteweg and de Vries $(\mathrm{KdV})$ equation where the environmental effect will be given as a perturbation term of the $\mathrm{KdV}$ equation. The environmental efects taken into considérations are river discharge and bottom sediment. The result show that a river discharge that flow opposite to wave motion will give the damping effect that is decreasing of the wave velocity and the amplitude. The bottom sediments will give the damping effect so that a fine bottom sediment would cause the waves propagte slower than coarse sediment.
\end{abstract}

\section{Introduction}

Tidal bore is very unique phenomena. This is a huge wave with the amplitude up to $5 \mathrm{~m}$ propagate from the mouth of estuary to the upstream with very fast velocity. The wave is called tsunami in the river. There only less than twenty of estuary have tidal bore phenomena in the world. One of them occure in Kampar River, Sumatra Indonesia. The waves ussually occure in November every years. This is generated by interaction of storm surge from the South china sea and river discharge at low tide condition. This wave have a local name the Bono and propagate with the velocity about more than $10 \mathrm{~m} / \mathrm{s}$.

The Kampar river is a convergent alluvial estuaries. The most of studies tidal bore in the avlluvial estuaries have been focused on a small-scale bore processes i.e the propagation zone. In this scale, the environmental effect can not be ignored. The tidal bore will be influenced by the environmental condition such as sediment resuspension, bed erosion and score [1-3]. Another hand, the environment can influence the tidal bore propagation through the damping effect or breaking of the tidal bore. For example the river discharge damps the tidal bore propagation in alluvial estuary [4]. Another observation showed that the suspended sediment concentration (SSC) will increase when the tidal bore passage [5]. When the bore propagate into the upstream, the free surface increase and flow velocity decrease rapidly under the bore front to satisfy the conservation of mass and energy. The present of bottom sediment will break the conservation of energy then the damping effect may occur. In the paper, the environmental effect such as river discharge and bottom

* Corresponding author: mubarak@unri.ac.id 
sediment on the tidal bore propagation are investigated. The study of environmental effect of this wave can help to identify dangerous region and can be used to river management plan. The paper is organized as follow, the physics of study of Kampar river and methodology are described in Sec-2. The tidal bore propagation and its environmental effect will be developed in Sec-3 and followed by the discussion of its simulation. The paper will be ended by a conclusion.

\section{Methodology}

Methodology used in the research is the simulations with analytical modeling supplied with the field observation.

\subsection{Fields Observation}

First of all, the morphology and a tidal bore observation of Kampar river will be highlighted. The Kampar river is a big river in Riau province Indonesia. It have $24.548 \mathrm{~km}^{2}$ area with the depth is around $7.8 \mathrm{~m}$ and the average depth is $413 \mathrm{~m}$. The river also used as main transportation with a groove of voyage crowded. From geomorphological point of view, the river is part of alluvial estuary that have trumpert form (converent) where this is a prerequest for the exixtence of a tidal bore. The tidal amplitude is around 2.1 to $4.5 \mathrm{~m}$ and propagate until $229 \mathrm{~km}$ along. The study area is depicted in Fig. 1.

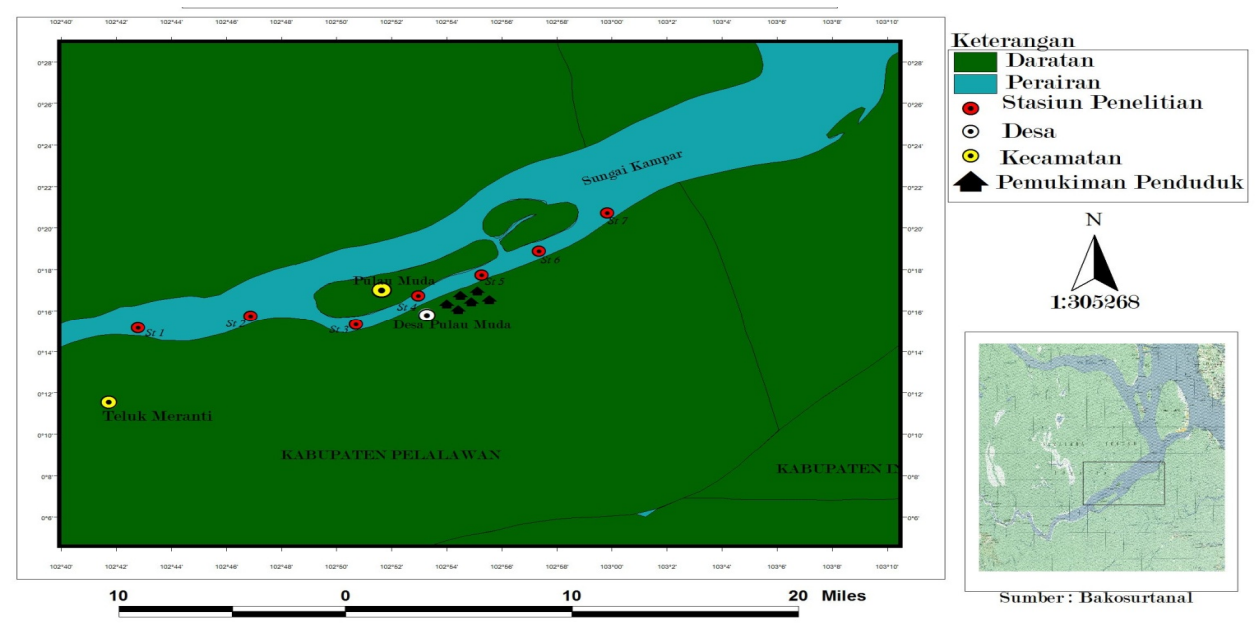

Fig.1. The study area. The red dot represent an observation point. The river water is represented by blue color.

The Kampar river have high river discharge and the interaction with the storm surge from the South china sea will generate a high amplitude of a tidal bore. The tidal bore in Kampar river ussualy occuring in November and December every years. The tidal bore wavefront can be seen in Fig.2. When the tidal bore passage, the local area of the river will be flooding due to run up of the waves. This is depicted in Fig.3. The picture describe a flooding during the tidal bore passage. The location is a small village called Teluk Meranti The run up can spread as far as $500 \mathrm{~m}$ from the river. At the sometime, the water calor is brown and very turbid. The animal form the river such as fish, snake and the other can just took to the land. 


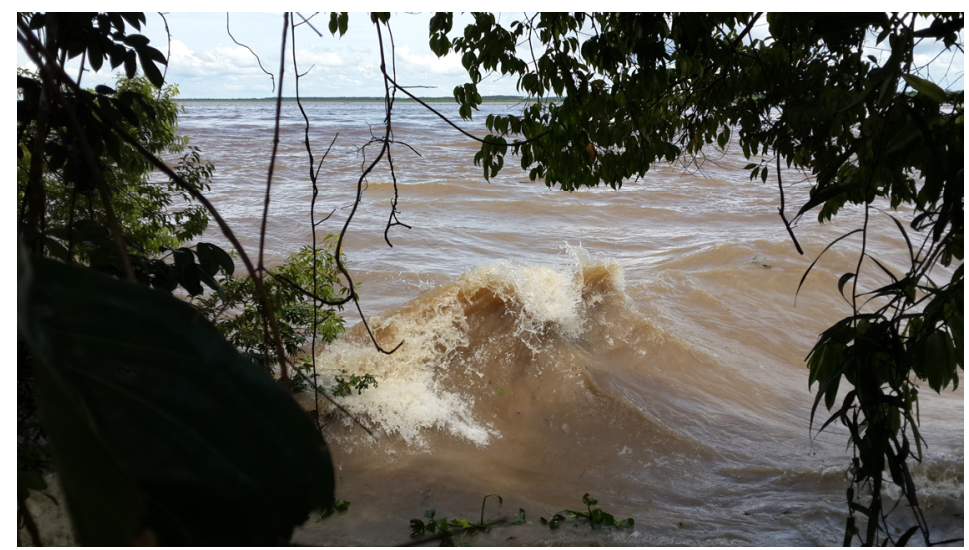

Fig.2. Tidal bore of the Kampar river on November, 2014 (courtesy: Wisnu A. Martono).

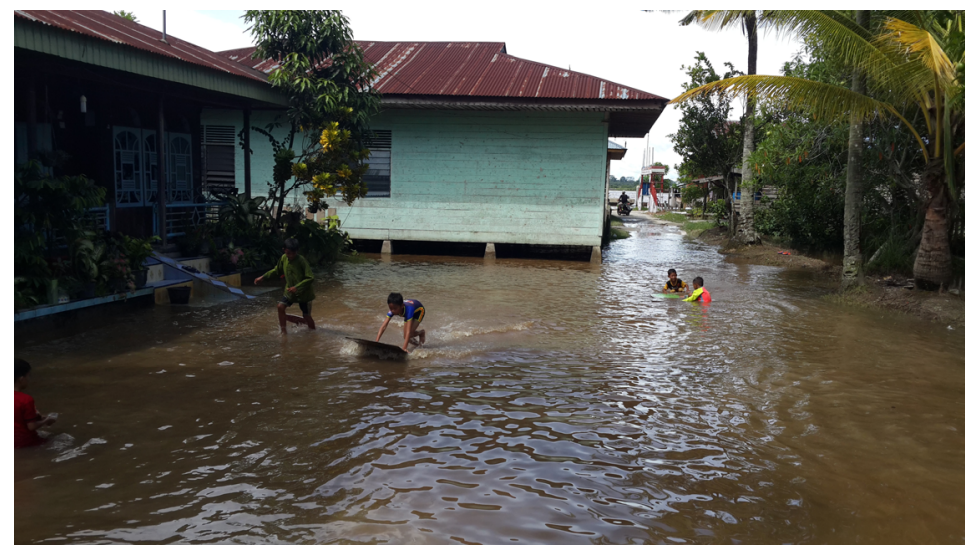

Fig.3. The flooding in the side of river when the tidal bore passage.

The measurement of bottom sedimen is depicted in Fig.4. The dominant bottom sedimen is mud sediment. The previous studies showed that the suspended sediment will resuspend and increase the erosion when the tidal bore passage [4]. For example, the sediment transport due to the tidal wave propagation created some smal island in the river and bank erosion near Muda island and mouth of Sekap river [5]. The measurement suspended sediment concentration (SSC) transport induced by tidal bore in the See reiver showed that $\mathrm{SSC}$ increase greater than $30 \mathrm{~g} / \mathrm{L}$ during the tidal ore passage. After the tidal bore passage, then the SSC gradually increase over the whole water column with the amplitude is about $2 \mathrm{~m}[5]$.

\subsection{Analytical Modeling}

Tidal bore in Kampar river appear in several form, sometime in the single pulse (undular bore) form and many pulse form. The first pulse have the amplitude higher than the succeses wave. The wave is not only propagate the energy but also the mass, so that this can transport the sediment. When the Bono propagate, there will be the silting-up of the bottom topography in many area of watershed especially in around Muda island and the 
estuary of Serkap river. Beside the sediment transport, the wave also cause the changing of the water colomn so that it can influence the river ecosystem. It is importance to study the effect of tidal bore propagation related to the environmental effect.

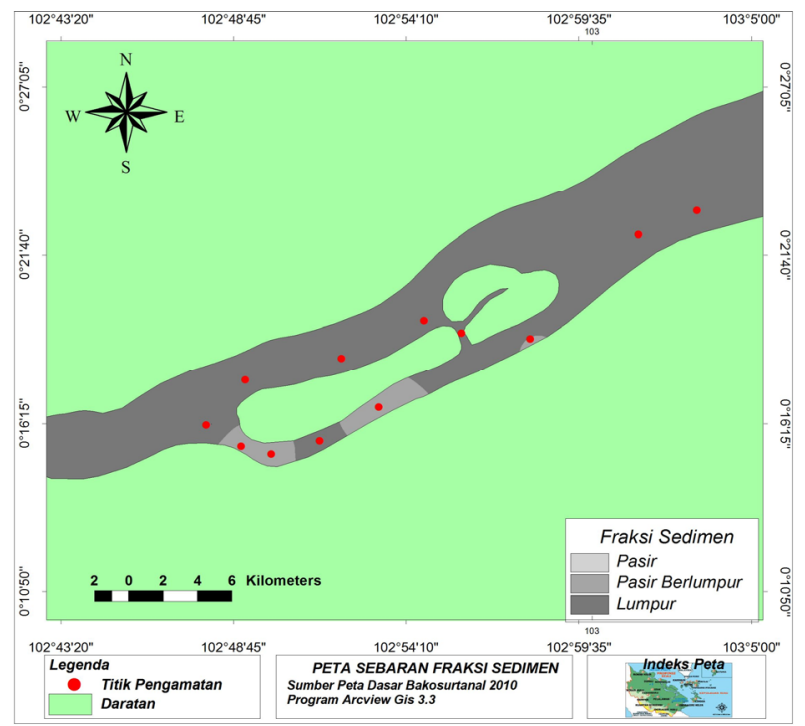

Fig.4. Botttom sediment observed June, 16 to June, 19 2016. The bottom sediment type is represented by grey color. The light grey color is sand, the medium grey is muddy sand and the heavy grey is a mud sediment.

The tidal bore is a nonlinear phenomena. This wave is a balance between nonlinear and dispersion effect. The most appropriate model for description of the tidal bore propagation is the Korteweg and De Vries $(\mathrm{KdV})$ equation. The derivation from this equation from shallow water model is very complicated. For simplicity we assume that the environment will give a perturbation for the tidal bore waves. This mean that the KdV equation should taking into account the environmental background. This phenomena can be described by the perturbed $\mathrm{KdV}$ equation as follow [8-10],

$$
\frac{\partial \eta}{\partial t}+(g h)^{\frac{1}{2}}\left(1+\frac{3}{2} \sqrt{g h} \frac{\eta}{h}\right) \frac{\partial \eta}{\partial x}+\frac{h^{2}}{6}(g h)^{\frac{1}{2}} \frac{\partial^{3} \eta}{\partial x^{3}}+F(x) \eta=0
$$

where $\eta$ tidal bore amplitude, $g$ acceleration of gravitation, $h$ is a water depth, $x$ and $t$ are spatial and temporal coordinate respectively and $F(x)$ is an environmental effect. The environmental effect of tidal bore propagation be investigated by giving the special form at $F(x)$ in the Equation (1). The single soliton solution will be used in this investigation. The first let we discuss the river discharge. The environmental effect such as river discharge also have significant contribution to damping effect of the tidal bore propagation [4]. The study showed that the fresh water discharge can no longer be neglected and this damp of tidal wave propagation significantly. The river discharge can seen as a constant perturbation so that in the Equation (1), we take $F(x)=\mu$, where $\mu$ is a constant parameter associated with river discharge. The single soliton solution of Equation (1) is given by [11],

$$
\eta(x, t)=8 \eta_{0}^{2} \exp \left[-\frac{8}{9} \mu \alpha\left(\frac{2 \alpha}{3 \beta}\right)^{\frac{1}{2}} t\right] \sec ^{2}(\varsigma)
$$


Where

$$
\begin{aligned}
& \zeta=\eta_{0} \exp \left[\frac{-4}{9} \mu \alpha\left(\frac{2 \alpha}{3 \beta}\right)^{1 / 2} t\right]\left[\left(\frac{2 \alpha}{3 \beta}\right)^{1 / 2}(x-V t)-\xi\right] \\
& \xi=3 \mu \eta_{0}^{2}\left(1-\exp \left[\frac{-8}{9} \mu \alpha\left(\frac{2 \alpha}{3 \beta}\right)^{1 / 2} t\right]\right) .
\end{aligned}
$$

By an assumption that the bottom sediment act as a constant force then the solution of Equation (1) is given by,

$$
\begin{gathered}
\eta(x, t)=2 \eta_{0}^{2} e^{\left(-\frac{3}{4} F_{0} t\right)} \sec h^{2}\left[\frac{\left(x+V_{d} t\right)}{\Delta}\right] \\
V_{d}=\left(1-\frac{\eta_{0}}{2 h}\right) \sqrt{g h}\left(1-e^{\left(-\frac{3}{4} F_{d} t\right)}\right) \quad ; \quad \Delta=\sqrt{\frac{4 h^{3}}{3 \eta_{0}}} e^{\frac{2}{3} F_{0} t}
\end{gathered}
$$

\section{Result and Discussion}

We will discuss two kind of the environmental effect, the river discharge and the sediment resuspension. In this paper, the tidal bore will be modeled as a single soliton. If tidal bore propagate from right (mouth of estuary) to left (upstream) and the river discharge flow from the upstream to mouth of estuary then the traveling wave parameters shoulfd be in the form of $x+V t$. The tidal bore propagation with the varying of river discharges are depicted in Fig.5.
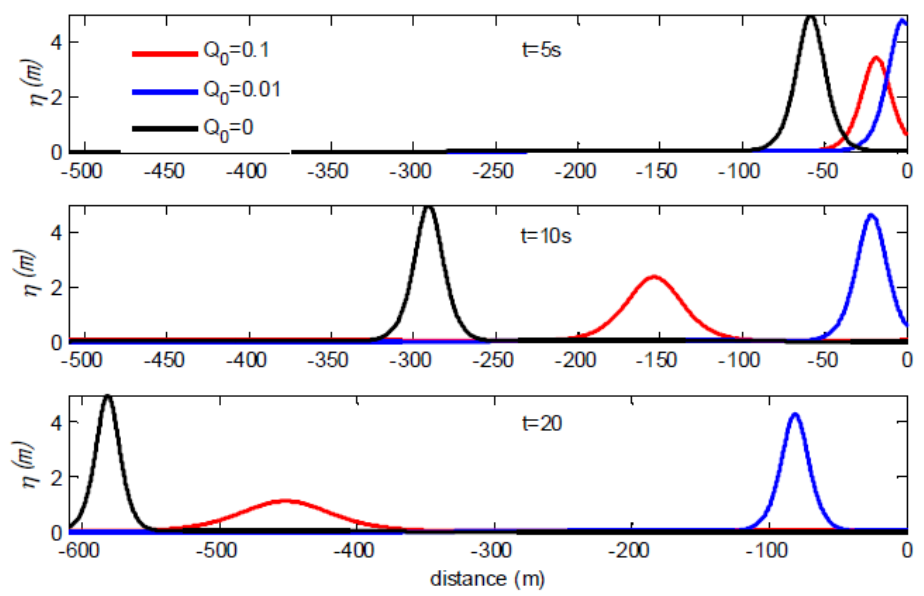

Fig.5. Simulation of tidal bore propagation with the varying river dischage.

This figure show various river effect on tidal bore propagation. This result show that the high discharge with propagate opposite with the propagation of tidal bore will damp the wave. The discharge will decrease the velocity and the amplitude of the tidal bore. The 
wave with high discharge will propagate faster but decrease the amplitude quickly compare with the lower discharge effect. The result also confirm by Bennenton [4].

The effect of bottom sediment respect to tidal bore propagtion can be studied through a definition of the bottom shear stress. The ottom stress ussualy define as $\tau=C_{D} \rho\left|V_{b}\right| V_{b}$, where $C_{D}$ is a drag coefficient and $V_{b}$ is bottom orbital velocity. Numerical study showed that the tidal bore will be damped due to the muddy bottom sediment and the wave dissipation causes significant downwave diffraction effects [12]. In this paper, we discuss the effect of bottom topography to the tidal bore propagation. Simulation of the solution by using various constant damping are depicted in Fig.5. The result show that the bottom sediment will give the damping effct of the tidal bore propagation. The tidal bore which propagate in the more dense bottom sediment will move faster than the thin sediment but the wave form more quickly decay. The bottom sediment in Kampar river compose of sand so that the damping effect is little paid influence on the wave propagation. This condition can explain that why the tidal bore on Kampar river can propagate over very long distance more than $200 \mathrm{~km}$.
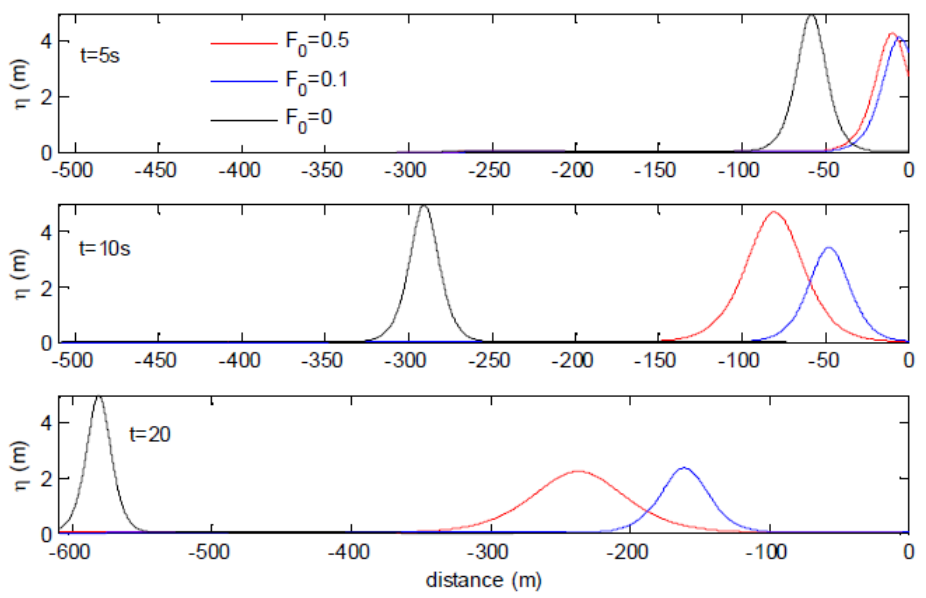

Fig.6. The tidal bore propagation with bottom sediment effect. The mud effect is modelled by assuming constant force. The 0.1 is represented by the blue color and 0.5 that is represented by red color.

\section{Conclusion}

Environmental effect of tidal bore propagation in the Kampar river is investigated. Two environmental effect such as river discharge and bottom sediment were studied. The result show that the high discharge with propagate opposite with the tidal bore propagation will damp the wave. The discharge will decrease the velocity and the amplitude of the tidal bore. The sediment effect show that the bottom sediment will give the damping effect of the tidal bore propagation. The simulation showed that the bore propagate in the dense bottom sediment will move faster than the thin sediment but the wave form more quickly decay.

This research founded by Universitas Riau in fiskal years 2016. 


\section{References}

[1] Chanson, H., Environmental, Ecological and Cultural Impacts of Tidal Bores, Benaks, Bonos and Burros. Proc. International Workshop on Environmental Hydraulics IWEH09, Theoretical, Experimental and Computational Solutions, Valencia, Spain, 29-30 Oct., Editor P.A. Lopez-Jimenez et al., Invited keynote lecture, 20 pages, (2009)

[2] Chanson, H., Current knowledge in hydraulic jumps and related phenomena. A survey of experimental results, European Journal of Mechanics Fluids 28 (2): 191-210, (2009)

[3] Chanson, H., Momentum considerations in hydraulic jumps and bores, J. Irrigation and Drainage Engineering, ASCE,138(4), 382, (2012)

[4] Bonennton, P., Andrea G. Filippini., L. Arpaia., N. Bonneton, and M. Ricchiuto, On nonlinear shoaling properties of enhanced Boussinesq models, J. Estuarine, Coastal and Shelf Science, 172, 121-127, (2016)

[5] Furgerot, L., D. Mouaze., B. Tessier., L. Perez., S. Haquin., P. Weill and A. Crave, Sediment transport induced by tidal bores. An estimation from suspended matter measurements in the Sée River (Mont-Saint-Michel Bay, northwestern France), Comptes Rendus Geoscience, 348 (6), 432-441, (2016)

[6] Chanson, H., Tidal bore in the North Branch of the Changjiang Estuary, Proc. International Conference on Estuaries and Coast, Hanzhou, China, (2003)

[7] Yulistianto, B., Fenomena Gelombang Pasang Bono di Muara Sungai Kampar, Dinamika TEKNIK SIPIL, 9(1), 19 - 26, (2009)

[8] Dingermans, M, Water Waves Propagation over uneven Bottoms, World Scientific, Singapore, (1997)

[9] Kundu, A., Tsunami and Nonlinear Waves, Springer Verlag, Berlin, (2007)

[10] Sulaiman, A., On the Internal soliton propagation over slope shelf topography, Int. Theoretical Physics and Nonlienar Phenomena Conf, Jakarta (2016)

[11] Sulaiman,A and M. Sadly, On Nonlinear Internal waves Propagation in the Narrow Strait of the Indonesian Seas, The 2nd Indonesia Japan Joint Scientific Symposium, Jakarta, (2006)

[12]Kaihatu,J.M., A. Sheremet, K.T. Holland., A model for the propagation of nonlinear surface waves over viscous muds, Coastal Engineering, 54, 752-764, (2007) 